ALEA, Lat. Am. J. Probab. Math. Stat. 14, 613-629 (2017)

DOI: 10.30757/ALEA.v14-30

\title{
Parameter Estimation of Complex Fractional Ornstein-Uhlenbeck Processes with Fractional Noise
}

\author{
Yong Chen, Yaozhong Hu and Zhi Wang \\ School of Mathematics, Hunan University of Science and Technology \\ Xiangtan, 411201, Hunan, China. \\ E-mail address: zhishi@pku.org.cn \\ Department of Mathematical and Statistical Sciences \\ University of Alberta, Edmonton, Alberta, Canada T6G 2G1 \\ E-mail address: yaozhong@ualberta.ca \\ School of Sciences, Ningbo University of Technology \\ Ningbo 315211, Zhejiang, China. \\ E-mail address: wangzhi1006@hotmail.com
}

\begin{abstract}
We obtain strong consistency and asymptotic normality of a least squares estimator of the drift coefficient for complex-valued Ornstein-Uhlenbeck processes disturbed by fractional noise, extending the result of $\mathrm{Hu}$ and Nualart (2010) to a special 2-dimensions. The strategy is to exploit the Garsia-RodemichRumsey inequality and complex fourth moment theorems. The main ingredients of this paper are the sample path regularity of a multiple Wiener-Itô integral and two equivalent conditions of complex fourth moment theorems in terms of the contractions of integral kernels and complex Malliavin derivatives.
\end{abstract}

\section{Introduction}

To model the Chandler wobble, or variation of latitude conerning with the rotation of the earth, M. Arató, A. N. Kolmogorov and Y. G. Sinai (see also Arató, 1982) proposed in the paper Arató et al. (1962) the following stochastic linear equation

$$
\mathrm{d} Z_{t}=-\gamma Z_{t} \mathrm{~d} t+\sqrt{a} \mathrm{~d} \zeta_{t}, \quad t \geq 0
$$

Received by the editors January 26th, 2017; accepted July 18th, 2017.

2010 Mathematics Subject Classification. 60H07, 60F05, 62M09.

Key words and phrases. Complex Wiener-Itô multiple integral, fractional Brownian motion, fractional Ornstein-Uhlenbeck process, fourth moment theorems, strong consistency, asymptotic normality.

Research supported by China Scholarship Council (201608430079), Simons Foundation (209206), Mathematical Tianyuan Foundation of China (11526117), Hubei Provincial NSFC (2016CFB526) and Zhejiang Provincial NSFC (LQ16A010006). 
where $Z_{t}=X_{1}(t)+\mathrm{i} X_{2}(t)$ is a complex-valued process, $\gamma=\lambda-\mathrm{i} \omega, \lambda>0, a>0$ and $\zeta_{t}$ is a complex Brownian motion. It is also suggested in Arató (1982) that the Brownian motion in (1.1) may be replaced by other processes. In this paper we consider the statistical estimator of $\gamma$ when the complex Brownian motion $\zeta$ in (1.1) is replaced by a complex fractional Brownian motion $\zeta_{t}=\frac{B_{t}^{1}+i B_{t}^{2}}{\sqrt{2}}$, where $\left(B_{t}^{1}, B_{t}^{2}\right)$ is a two dimensional fractional Brownian motion with $H \in\left[\frac{1}{2}, \frac{3}{4}\right)$. [We shall fix the Hurst parameter and then omit the explicit dependence of the process on the Hurst parameter.] From now on we assume that $\zeta$ is a complex fractional Brownian motion of Hurst parameter $H \in(1 / 2,3 / 4)$.

To compare with the work in Hu and Nualart (2010), we write (1.1) as

$$
\left[\begin{array}{l}
\mathrm{d} X_{1}(t) \\
\mathrm{d} X_{2}(t)
\end{array}\right]=\left[\begin{array}{cc}
-\lambda & -\omega \\
\omega & -\lambda
\end{array}\right]\left[\begin{array}{l}
X_{1}(t) \\
X_{2}(t)
\end{array}\right] \mathrm{d} t+\sqrt{\frac{a}{2}}\left[\begin{array}{l}
d B_{t}^{1} \\
d B_{t}^{2}
\end{array}\right]
$$

Thus (1.1) can be considered as a particular two dimensional Langevin equation driven by fractional Brownian motions. However, we find it is more convenient to use the complex valued equation (1.1).

Motivated by the work of $\mathrm{Hu}$ and Nualart (2010), we also consider a least squares estimator for $\gamma$. To this end, we intuitively rewrite (1.1) as

$$
\dot{Z}_{t}+\gamma Z_{t}=\sqrt{a} \dot{\zeta}_{t}, \quad 0 \leq t \leq T .
$$

We minimize $\int_{0}^{T}\left|\dot{Z}_{t}+\gamma Z_{t}\right|^{2} \mathrm{~d} t$ to obtain a least squares estimator of $\gamma$ as follows.

$$
\hat{\gamma}_{T}=-\frac{\int_{0}^{T} \bar{Z}_{t} \mathrm{~d} Z_{t}}{\int_{0}^{T}\left|Z_{t}\right|^{2} \mathrm{~d} t}=\gamma-\sqrt{a} \frac{\int_{0}^{T} \bar{Z}_{t} \mathrm{~d} \zeta_{t}}{\int_{0}^{T}\left|Z_{t}\right|^{2} \mathrm{~d} t} .
$$

The main results of the present paper are the strong consistency and the asymptotic normality of the estimator $\hat{\gamma}_{T}$ which we state as follows.

Theorem 1.1. Let $H \in\left[\frac{1}{2}, \frac{3}{4}\right)$.

(i) The least squares estimator $\hat{\gamma}_{T}$ is strongly consistent. Namely, $\hat{\gamma}_{T}$ converges to $\gamma$ almost surely as $T \rightarrow \infty$.

(ii) $\sqrt{T}\left(\hat{\gamma}_{T}-\gamma\right)$ is asymptotically normal. Namely,

$$
\begin{aligned}
& \sqrt{T}\left[\hat{\gamma}_{T}-\gamma\right] \stackrel{\text { law }}{\rightarrow} \mathcal{N}\left(0, \frac{1}{2 d^{2} a} \mathrm{C}\right) \quad \text { as } T \rightarrow \infty, \\
\text { where } \mathrm{C}= & {\left[\begin{array}{cc}
\sigma^{2}+c & b \\
b & \sigma^{2}-c
\end{array}\right] \text { with } } \\
\sigma^{2}= & \frac{2}{\Gamma(2-2 H)^{2}} \int_{[0, \infty)^{2}} \mathrm{~d} x \mathrm{~d} y \frac{(x y)^{1-2 H}}{(x+y)(x+\bar{\gamma})(y+\gamma)} \\
& +\frac{\Gamma^{2}(2 H-1)}{2 \lambda}\left(\frac{2}{|\gamma|^{4 H-2}}+\frac{1}{\gamma^{4 H-2}}+\frac{1}{\bar{\gamma}^{4 H-2}}\right) \\
c+\mathrm{i} b= & \frac{2}{\Gamma(2-2 H)^{2}} \int_{[0, \infty)^{2}} \frac{(x y)^{1-2 H}}{(y+\gamma)^{2}}\left[\frac{1}{x+y}+\frac{1}{x+\gamma}\right] \mathrm{d} x \mathrm{~d} y . \\
d= & \frac{\Gamma(2 H-1)}{2 \lambda}\left(\frac{1}{\gamma^{2 H-1}}+\frac{1}{\bar{\gamma}^{2 H-1}}\right) .
\end{aligned}
$$


In the special case when $H=\frac{1}{2}$, we have

$$
\sqrt{T}\left[\hat{\gamma}_{T}-\gamma\right] \stackrel{\text { law }}{\rightarrow} \mathcal{N}\left(0, \frac{\lambda}{4 a} \operatorname{Id}_{2}\right)
$$

where $\mathrm{Id}_{2}$ is a $2 \times 2$ identity matrix.

Remark 1.2. An important new feature for the case of fractional OrnsteinUhlenbeck process $(H \in(1 / 2,3 / 4))$ is that the limiting distribution is no longer independent Gaussian as in the case of Brownian motion case $(H=1 / 2)$. We will discuss exclusively the case $H \neq 1 / 2$ since the case $H=1 / 2$ is easy.

A minor difference between the case of one dimensional fractional Ornstein-Uhlenbeck process considered in Hu and Nualart (2010) and our complex case is that in our least squares estimator $\hat{\gamma}$ defined by (1.3), we have $\int_{0}^{T} \bar{Z}_{t} \mathrm{~d} Z_{t}$ in the numerator, while in $\mathrm{Hu}$ and Nualart (2010) it is $\int_{0}^{T} X_{t} \mathrm{~d} X_{t}$. However, this minor difference causes a big unpleasant trouble. By using Itô formula the latter is expressed as $X_{T}^{2}$ plus another manageable term. This is critical in the proof of the strong consistency of the estimator since it allows us to use a famous theorem of Pickands in Hu and Nualart (2010). However, we cannot no longer apply the Itô formula to $\int_{0}^{T} \bar{Z}_{t} \mathrm{~d} Z_{t}$ to obtain a similar identity. To get around this difficulty we shall use another famous result, the Garsia-Rodemich-Rumsey inequality, see e.g. Hu (2017, Theorem 2.1).

To show the asymptotic normality, we may use a multi-dimensional fourth moment theorem. However, we develop a complex version of the fourth moment theorem which is easier to use in our case. To state the theorem we denote $\alpha_{H}=H(2 H-1)$ and $\phi(s, t)=\alpha_{H}|s-t|^{2 H-2}$ and define the Hilbert space

$$
\mathfrak{H}:=L_{\phi}^{2}=\left\{\left.f\left|f: \mathbb{R}_{+} \rightarrow \mathbb{R},\right| f\right|_{\phi} ^{2}:=\int_{0}^{\infty} \int_{0}^{\infty} f(s) f(t) \phi(s, t) \mathrm{d} s \mathrm{~d} t<\infty\right\} .
$$

Now the theorem is stated as follows.

Theorem 1.3 (Fourth Moment Theorems). Let $\left\{F_{k}=I_{m, n}\left(f_{k}\right)\right\}$ with $f_{k} \in \mathfrak{H}_{\mathbb{C}}^{\odot m} \otimes$ $\mathfrak{H}_{\mathbb{C}}^{\odot n}$ be a sequence of $(m, n)$-th complex Wiener-Itô multiple integrals (see the next section for a discussion), with $m$ and $n$ fixed and $m+n \geq 2$. Suppose that as $k \rightarrow \infty, \mathbb{E}\left[\left|F_{k}\right|^{2}\right] \rightarrow \sigma^{2}$ and $\mathbb{E}\left[F_{k}^{2}\right] \rightarrow c+\mathrm{i} b$, where $|\cdot|$ is the absolute value (or modulus) of a complex number and $c, b \in \mathbb{R}$. Then the following statements are equivalent:

(i) The sequence $\left(\operatorname{Re} F_{k}, \operatorname{Im} F_{k}\right)$ converges in law to a bivariate normal distribution with covariance matrix $\mathrm{C}=\frac{1}{2}\left[\begin{array}{cc}\sigma^{2}+c & b \\ b & \sigma^{2}-c\end{array}\right]$,

(ii) $\mathbb{E}\left[\left|F_{k}\right|^{4}\right] \rightarrow c^{2}+b^{2}+2 \sigma^{4}$.

(iii) $\left\|f_{k} \otimes_{i, j} f_{k}\right\|_{\mathfrak{H}^{\otimes(2(l-i-j))}} \rightarrow 0$ and $\left\|f_{k} \otimes_{i, j} h_{k}\right\|_{\mathfrak{H} \otimes(2(l-i-j))} \rightarrow 0$ for any $0<$ $i+j \leq l-1$ where $l=m+n$ and $h_{k}$ is the kernel of $\bar{F}_{k}$, i.e., $\bar{F}_{k}=I_{n, m}\left(h_{k}\right)$.

(iv) $\left\|f_{k} \tilde{\otimes}_{i, j} f_{k}\right\|_{\mathfrak{H}^{\otimes(2(l-i-j))}} \rightarrow 0$ and $\left\|f_{k} \tilde{\otimes}_{i, j} h_{k}\right\|_{\mathfrak{H}^{\otimes(2(l-i-j))}} \rightarrow 0$ for any $0<$ $i+j \leq l-1$.

(v) $\left\|D F_{k}\right\|_{\mathfrak{H}}^{2},\left\|D \bar{F}_{k}\right\|_{\mathfrak{H}}^{2}$ and $\left\langle D F_{k}, D \bar{F}_{k}\right\rangle_{\mathfrak{H}}$ converge to a constant in $L^{2}(\Omega)$ as $k$ tends to infinity, where $D$ is the complex Malliavin derivatives. That is to say, $\operatorname{Var}\left(\left\|D F_{k}\right\|_{\mathfrak{H}}^{2}\right) \rightarrow 0, \operatorname{Var}\left(\left\|D \bar{F}_{k}\right\|_{\mathfrak{H}}^{2}\right) \rightarrow 0$ and $\operatorname{Var}\left(\left\langle D F_{k}, D \bar{F}_{k}\right\rangle_{\mathfrak{H}}\right) \rightarrow 0$ as $k$ tends to infinity. 


\section{Remark 1.4.}

1) If $m=n$ and $\mathbb{E}\left[F_{k}^{2}\right]=0$ or if $m \neq n$, then $C=\frac{\sigma^{2}}{2} \operatorname{Id}_{2}$. That is to say, the limit is a complex Gaussian variable $\mathcal{C N}\left(0, \sigma^{2}\right)$. Theorem 7 of Nualart and Ortiz-Latorre (2008) is concerning multi-dimensional fourth moment theorems, but it requires $C=\frac{\sigma^{2}}{2} I d$. Thus, our results are more general.

2) We shall give a different and simpler proof of the theorem in next section. The equivalence (i) $\Leftrightarrow$ (ii) is shown by an indirect method in Chen and Liu (2017) and by Stein's method in Campese (2015). In this paper, we show that (i) $\Rightarrow$ (ii) $\Rightarrow$ (iii) $\Rightarrow$ (iv) $\Rightarrow$ (v) $\Rightarrow$ (i) directly. We make use of (iii) to show the asymptotic normality which is simpler than to use (v) as in previous work of $\mathrm{Hu}$ and Nualart (2010). In addition, similar to the realvalued case, see e.g. Hu (2017, p169), one can show (iv) $\Rightarrow$ (iii) directly, see Chen and Jiang (2017).

\section{Preliminaries: complex multiple Wiener-Itô integrals}

Denote by $\left(B_{t}, t \geq 0\right)$ a $\mathrm{fBm}$ of Hurst parameter $H \in(1 / 2,3 / 4)$. Then a Gaussian isonormal process associated with $\mathfrak{H}$ is given by Wiener integrals with respect to a $\mathrm{fBm}$ for any deterministic kernel $\in \mathfrak{H}$ (where $\mathfrak{H}$ is defined by (1.9)):

$$
B(f)=\int_{0}^{\infty} f(s) \mathrm{d} B_{s}, \quad \forall f \in \mathfrak{H} .
$$

Let $\tilde{B}(\cdot)$ be an independent copy of the fractional Brownian motion $B(\cdot)$. Following the same idea of Chen and Liu (2017), we define complex Gaussian isonormal processes and complex multiple Wiener-Itô integrals with respect to fBm as follows. For any $f=f_{1}+\mathrm{i} f_{2}$ with $f_{1}, f_{2} \in \mathfrak{H}$, define that

$$
\begin{aligned}
\mathfrak{H}_{\mathbb{C}} & :=\left\{f_{1}+\mathrm{i} f_{2}: f_{1}, f_{2} \in \mathfrak{H}\right\}, \quad\left\langle f_{1}+\mathrm{i} f_{2}, f_{1}+\mathrm{i} f_{2}\right\rangle_{\mathfrak{H}}=\left\langle f_{1}, f_{1}\right\rangle_{\mathfrak{H}}+\left\langle f_{2}, f_{2}\right\rangle_{\mathfrak{H}}, \\
B(f) & =B\left(f_{1}\right)+\mathrm{i} B\left(f_{2}\right), \quad \zeta(f)=\frac{1}{\sqrt{2}}[B(f)+\mathrm{i} \tilde{B}(f)] .
\end{aligned}
$$

Then $\zeta$ is called a complex isonormal Gaussian process over $\mathfrak{H}_{\mathbb{C}}$, which is a centered complex Gaussian family satisfying

$$
\mathbb{E}\left[\zeta(h)^{2}\right]=0, \quad \mathbb{E}[\zeta(g) \overline{\zeta(h)}]=\langle g, h\rangle_{\mathfrak{H}_{\mathfrak{C}}}, \quad \forall g, h \in \mathfrak{H}_{\mathbb{C}} .
$$

From now on, without ambiguity, we still denote $\mathfrak{H}_{\mathbb{C}}$ by $\mathfrak{H}$.

Definition 2.1 (Complex multiple Wiener-Itô integrals). For a fixed $(p, q)$, suppose that $g \in \mathfrak{H}^{\odot p} \otimes \mathfrak{H}^{\odot q}$, we call $I_{p, q}(g)$ the complex multiple Wiener-Itô integral of $g$ with respect to $\zeta$, see Chen and Liu (2017). And if $f \in \mathfrak{H}^{\otimes(p+q)}$ then we define

$$
I_{p, q}(f)=I_{p, q}(\tilde{f}),
$$

where $\tilde{f}$ is the symmetrization of $f$ in the sense of Itô (1952):

$$
\tilde{f}\left(t_{1}, \ldots, t_{p+q}\right)=\frac{1}{p ! q !} \sum_{\pi} \sum_{\sigma} f\left(t_{\pi(1)}, \ldots, t_{\pi(p)}, t_{\sigma(1)}, \ldots, t_{\sigma(q)}\right),
$$

where $\pi$ and $\sigma$ run over all permutations of $(1, \ldots, p)$ and $(p+1, \ldots, p+q)$ respectively. 
It is easy to see that $\overline{I_{p, q}(f)}=I_{q, p}(\bar{f})$ and

$$
\mathbb{E}\left[I_{p, q}(f) \overline{I_{p^{\prime}, q^{\prime}}(g)}\right]=\delta_{p, p^{\prime}} \delta_{q, q^{\prime}} p ! q !\langle\tilde{f}, \tilde{g}\rangle, \quad \text { (Itô's isometry) }
$$

where the Kronecker delta $\delta_{p, p^{\prime}}$ is 1 when $p^{\prime}$ is equal to $p$, and is 0 otherwise, and $\langle\cdot, \cdot\rangle$ is the inner product on $\mathfrak{H}^{\otimes(p+q)}$. As a consequence,

$$
\mathbb{E}\left[\left|I_{p, q}(f)\right|^{2}\right]=p ! q !\|\tilde{f}\|^{2} \leq p ! q !\|f\|^{2}, \quad \text { (Itô's isometry). }
$$

The proof of Theorem 1.3 proceeds through several propositions and lemmas. Firstly, we define the contraction of $(i, j)$ indices of two symmetric functions.

Definition 2.2. For two symmetric functions $f \in \mathfrak{H}^{\odot p_{1}} \otimes \mathfrak{H}^{\odot q_{1}}, g \in \mathfrak{H}^{\odot p_{2}} \otimes \mathfrak{H}^{\odot q_{2}}$ and $i \leq p_{1} \wedge q_{2}, j \leq q_{1} \wedge p_{2}$, the contraction of $(i, j)$ index is defined as (see Chen, 2014)

$$
\begin{aligned}
& f \otimes_{i, j} g\left(t_{1}, \ldots, t_{p_{1}+p_{2}-i-j} ; s_{1}, \ldots, s_{q_{1}+q_{2}-i-j}\right) \\
& =\int_{\mathbb{R}_{+}^{2 l}} \mathrm{~d} \vec{u} \mathrm{~d} \overrightarrow{u^{\prime}} \phi\left(u_{1}, u_{1}^{\prime}\right) \ldots \phi\left(u_{i}, u_{i}^{\prime}\right) f\left(t_{1}, \ldots, t_{p_{1}-i}, u_{1}, \ldots, u_{i} ; s_{1} \ldots, s_{q_{1}-j}, v_{1} \ldots, v_{j}\right) \\
& \times g\left(t_{p_{1}-i+1}, \ldots, t_{p-l}, v_{1}^{\prime}, \ldots, v_{j}^{\prime} ; s_{q_{1}-j+1}, \ldots, s_{q-l}, u_{1}^{\prime}, \ldots, u_{i}^{\prime}\right) \\
& \times \phi\left(v_{1}, v_{1}^{\prime}\right) \ldots \phi\left(v_{j}, v_{j}^{\prime}\right) \mathrm{d} \vec{v} \mathrm{~d} \overrightarrow{v^{\prime}}
\end{aligned}
$$

where $l=i+j, p=p_{1}+p_{2}, q=q_{1}+q_{2}, \vec{u}=\left(u_{1}, \cdots, u_{i}\right), \overrightarrow{u^{\prime}}=\left(u_{1}^{\prime}, \cdots, u_{i}^{\prime}\right)$ and $\vec{v}=\left(v_{1}, \cdots, v_{j}\right), \overrightarrow{v^{\prime}}=\left(v_{1}^{\prime}, \cdots, v_{j}^{\prime}\right)$.

By convention, $f \otimes_{0,0} g=f \otimes g$ denotes the tensor product of $f$ and $g$. We write $f \tilde{\otimes}_{p, q} g$ for the symmetrization of $f \otimes_{p, q} g$. In what follows, we use the convention $f \otimes_{i, j} g=0$ if $i>p_{1} \wedge q_{2}$ or $j>q_{1} \wedge p_{2}$.

Our next result is a technical lemma.

Lemma 2.3. Suppose that $F=I_{m, n}(f)$ with $f \in \mathfrak{H}^{\odot m} \otimes \mathfrak{H}^{\odot n}$ and that $\bar{F}=I_{n, m}(h)$. Then

$$
\begin{aligned}
& \mathbb{E}\left[|F|^{4}\right]-2\left(\mathbb{E}\left[|F|^{2}\right]\right)^{2}-\left|\mathbb{E}\left[F^{2}\right]\right|^{2} \\
& =\sum_{0<i+j<l}\left(\begin{array}{c}
m \\
i
\end{array}\right)^{2}\left(\begin{array}{c}
n \\
j
\end{array}\right)^{2}(m ! n !)^{2}\left\|f \otimes_{i, j} f\right\|_{\mathfrak{H} \otimes(2(l-i-j))}^{2}+\sum_{r=1}^{l-1}((l-r) !)^{2}\left\|\psi_{r}\right\|_{\mathfrak{H} \otimes(2(l-r))}^{2} \\
& =\sum_{0<i+j<l}\left(\begin{array}{c}
m \\
i
\end{array}\right)\left(\begin{array}{c}
n \\
i
\end{array}\right)\left(\begin{array}{c}
n \\
j
\end{array}\right)\left(\begin{array}{c}
m \\
j
\end{array}\right)(m ! n !)^{2}\left\|f \otimes_{i, j} h\right\|_{\mathfrak{H} \otimes(2(l-i+j))}^{2} \\
& +\sum_{r=1}^{l-1}(2 m-r) !(2 n-r) !\left\|\varphi_{r}\right\|_{\mathfrak{H} \otimes 2(l-r)}^{2},
\end{aligned}
$$

where $l=m+n$ and

$$
\begin{aligned}
\psi_{r} & =\sum_{i+j=r} i ! j !\left(\begin{array}{c}
m \\
i
\end{array}\right)^{2}\left(\begin{array}{c}
n \\
j
\end{array}\right)^{2} f \tilde{\otimes}_{i, j} h \\
\varphi_{r} & =\sum_{i+j=r} i ! j !\left(\begin{array}{c}
m \\
i
\end{array}\right)\left(\begin{array}{c}
n \\
i
\end{array}\right)\left(\begin{array}{c}
n \\
j
\end{array}\right)\left(\begin{array}{c}
m \\
j
\end{array}\right) f \tilde{\otimes}_{i, j} f .
\end{aligned}
$$


Proof: Applying Lemma 4.1 of Chen (2014) to $G=F$, we obtain that

$$
\begin{aligned}
& \mathbb{E}\left[|F|^{4}\right]-\left(\mathbb{E}\left[|F|^{2}\right]\right)^{2} \\
= & \sum_{i+j>0}\left(\begin{array}{c}
m \\
i
\end{array}\right)^{2}\left(\begin{array}{c}
n \\
j
\end{array}\right)^{2}(m ! n !)^{2}\left\|f \otimes_{i, j} f\right\|_{\mathfrak{H}^{\otimes(2(l-i-j))}}^{2}+\sum_{r=1}^{l}((l-r) !)^{2}\left\|\psi_{r}\right\|_{\mathfrak{H} \otimes(2(l-r))}^{2} .
\end{aligned}
$$

We calculate the term $\psi_{l}=f \otimes_{m, n} h$ :

$$
\begin{aligned}
f \otimes_{m, n} h= & \int_{\mathbb{R}_{+}^{m+n}} d \vec{u} d \overrightarrow{u^{\prime}} d \vec{v} d \overrightarrow{v^{\prime}} \phi\left(u_{1}, u_{1}^{\prime}\right) \ldots \phi\left(u_{m}, u_{m}^{\prime}\right) \phi\left(v_{1}, v_{1}^{\prime}\right) \ldots \phi\left(v_{n}, v_{n}^{\prime}\right) \\
& \times f\left(u_{1}, \ldots, u_{m} ; v_{1}, \ldots, v_{n}\right) h\left(v_{1}^{\prime}, \ldots, v_{n}^{\prime} ; u_{1}^{\prime}, \ldots, u_{m}^{\prime}\right) \\
= & \|f\|_{\mathfrak{H}^{\otimes(m+n)}}^{2}=\frac{1}{m ! n !} \mathbb{E}\left[|F|^{2}\right]
\end{aligned}
$$

where the last equality is from Ito's isometry (2.7). Next, we calculate the term $f \otimes_{m, n} f$ in Eq.(2.12) according to whether $m=n$ or not. We consider the case $m \neq n$ first. Without loss of generality we can take $m>n$. By Definition 2.2 we have that if $i>n$ or $j>n$ then $f \otimes_{i, j} f=0$. Therefore, if $m \neq n$ then

$$
f \otimes_{m, n} f=0=\mathbb{E}\left[F^{2}\right],
$$

where the last equality is Itô's isometry (2.6). If $m=n$, similarly to show (2.13), we obtain that

$$
f \otimes_{m, m} f=\langle f, h\rangle_{\mathfrak{H} \otimes(m+n)}=\frac{1}{(m !)^{2}} \mathbb{E}\left[F^{2}\right] .
$$

Substituting (2.15) or (2.14) according to whether $m=n$ or not, and (2.13), into (2.12), we obtain (2.8). Applying Lemma 4.1 of Chen (2014) to $G=\bar{F}$, we can show (2.9) similarly.

Remark 2.4. Both (2.8) and (2.9) are from the product formula and are analogous to (5.2.6) of Nourdin and Peccati (2012). One can also obtain another expansion of the moment analogous to (5.2.5) of Nourdin and Peccati (2012) if the complex Ornstein-Uhlenbeck operator is explored, see Chen and Jiang (2017).

Notation 1. Suppose that $f\left(\vec{t}^{m}, \vec{s}^{n}\right) \in \mathfrak{H}^{\odot m} \otimes \mathfrak{H}^{\odot n}$. Denote

$$
f_{u}\left(\vec{t}^{m-1}, \vec{s}^{n}\right)=f\left(\vec{t}^{m-1}, u, \vec{s}^{n}\right), \quad f^{v}\left(\vec{t}^{m}, \vec{s}^{n-1}\right)=f\left(\vec{t}^{m}, \vec{s}^{n-1}, v\right) .
$$

Clearly, $f_{u}\left(\vec{t}^{m-1}, \vec{s}^{n}\right) \in \mathfrak{H}^{\odot m-1} \otimes \mathfrak{H}^{\odot n}$ and $f^{v}\left(\vec{t}^{m}, \vec{s}^{n-1}\right) \in \mathfrak{H}^{\odot m} \otimes \mathfrak{H}^{\odot n-1}$.

Definition 2.5 (Complex Malliavin Derivatives). Let $\mathcal{S}$ denote the set of all random variables of the form

$$
f\left(\zeta^{H}\left(\varphi_{1}\right), \cdots, \zeta^{H}\left(\varphi_{m}\right)\right)
$$

where $f \in C_{\uparrow}^{\infty}\left(\mathbb{C}^{m}\right)$ and $\varphi_{i} \in \mathfrak{H}, i=1,2, \cdots, m$. Let $F \in \mathcal{S}$ be given by (2.17). The complex Malliavin derivative of $F$ is the element of $L^{2}(\Omega, \mathfrak{H})$ defined by:

$$
\begin{aligned}
& D F=\sum_{i=1}^{m} \partial_{i} f\left(\zeta^{H}\left(\varphi_{1}\right), \ldots, \zeta^{H}\left(\varphi_{m}\right)\right) \varphi_{i}, \\
& \bar{D} F=\sum_{i=1}^{m} \bar{\partial}_{i} f\left(\zeta^{H}\left(\varphi_{1}\right), \ldots, \zeta^{H}\left(\varphi_{m}\right)\right) \bar{\varphi}_{i},
\end{aligned}
$$


where $\partial_{j} f=\frac{\partial}{\partial z_{j}} f\left(z_{1}, \ldots, z_{m}\right), \quad \bar{\partial}_{j} f=\frac{\partial}{\partial \bar{z}_{j}} f\left(z_{1}, \ldots, z_{m}\right)$ are the Wirtinger derivatives, see e.g. Campese (2015).

Proposition 2.6. Suppose that $l=m+n$ and

$$
\begin{aligned}
& \eta_{r}=\sum_{i+j=r} i\left(\begin{array}{c}
m \\
i
\end{array}\right)^{2}\left(\begin{array}{c}
n \\
j
\end{array}\right)^{2} i ! j ! f \tilde{\otimes}_{i, j} h, \\
& \xi_{r}=\sum_{i+j=r} j\left(\begin{array}{c}
m \\
i
\end{array}\right)^{2}\left(\begin{array}{c}
n \\
j
\end{array}\right)^{2} i ! j ! h \tilde{\otimes}_{j, i} f, \\
& \nu_{r}=\sum_{i+j=r} i\left(\begin{array}{c}
m \\
i
\end{array}\right)\left(\begin{array}{c}
n \\
i
\end{array}\right)\left(\begin{array}{c}
n \\
j
\end{array}\right)\left(\begin{array}{c}
m \\
j
\end{array}\right) i ! j ! f \tilde{\otimes}_{i, j} f,
\end{aligned}
$$

then we have that

$$
\begin{aligned}
\operatorname{Var}\left(\left\|D I_{m, n}(f)\right\|_{\mathfrak{H}}^{2}\right) & =\sum_{r=1}^{l-1}[(l-r) !]^{2}\left\|\eta_{r}\right\|_{\mathfrak{H}^{\otimes(2(l-r))}}^{2}, \\
\operatorname{Var}\left(\left\|\bar{D} I_{m, n}(f)\right\|_{\mathfrak{H}}^{2}\right) & =\sum_{r=1}^{l-1}[(l-r) !]^{2}\left\|\xi_{r}\right\|_{\mathfrak{H}^{\otimes(2(l-r))}}^{2}, \\
\operatorname{Var}\left(\left\langle D I_{m, n}(f), D \overline{I_{m, n}(f)}\right\rangle_{\mathfrak{H}}\right) & =\sum_{r=1}^{l-1}(2 m-r) !(2 n-r) !\left\|\nu_{r}\right\|_{\mathfrak{H}^{\otimes(2(l-r))}}^{2} .
\end{aligned}
$$

Proof: We need only to show (2.23) since the other two are similar. Denote $l^{\prime}=$ $m+n-1$.

Step 1: Using product formula. By Theorem 12(D) of Itô (1952) and the product formula of complex Wiener-Itô multiple integrals (Theorem 3.2 of Chen, 2014), we have that

$$
\begin{aligned}
& \frac{1}{m^{2}}\left\|D \cdot\left(I_{m, n}(f)\right)\right\|_{\mathfrak{H}}^{2} \\
& =\left\|I_{m-1, n}\left(f_{u}\left(\vec{t}^{m-1}, \vec{s}^{n}\right)\right)\right\|_{\mathfrak{H}}^{2} \\
& =\int_{[0, \infty)^{2}} \mathrm{~d} u \mathrm{~d} v \phi(u, v) I_{m-1, n}\left(f_{u}\left(\vec{t}^{m-1}, \vec{s}^{n}\right)\right) \overline{I_{m-1, n}\left(f_{v}\left(\vec{t}^{m-1}, \vec{s}^{n}\right)\right)} \\
& =\sum_{i=0}^{m-1} \sum_{j=0}^{n}\left(\begin{array}{c}
m-1 \\
i
\end{array}\right)^{2}\left(\begin{array}{c}
n \\
j
\end{array}\right)^{2} i ! j ! \\
& \times \int_{[0, \infty)^{2}} \mathrm{~d} u \mathrm{~d} v \phi(u, v) I_{l^{\prime}-i-j, l^{\prime}-i-j}\left(f_{u}\left(\vec{t}^{m-1}, \vec{s}^{n}\right) \otimes_{i, j} h^{v}\left(\vec{t}^{n}, \vec{s}^{m-1}\right)\right)
\end{aligned}
$$

where $h^{v}\left(\vec{t}^{n}, \vec{s}^{m-1}\right)=\bar{f}_{v}\left(\vec{s}^{m-1}, \vec{t}^{n}\right)=\bar{f}\left(\vec{s}^{m-1}, v, \vec{t}^{n}\right)$ and

$$
\begin{aligned}
& \left(f_{u}\left(\vec{t}^{m-1}, \vec{s}^{n}\right) \otimes_{i, j} h^{v}\left(\vec{t}^{n}, \vec{s}^{m-1}\right)\right)\left(\vec{t}^{l^{\prime}-i-j}, u, \vec{s}^{l^{\prime}-i-j}, v\right) \\
& =\int_{[0, \infty)^{i+j}} \mathrm{~d} \vec{x}^{i} \mathrm{~d}{\overrightarrow{x^{\prime}}}^{i} \phi\left(x_{1}, x_{1}^{\prime}\right) \ldots \phi\left(x_{i}, x_{i}^{\prime}\right) f_{u}\left(\vec{t}^{m-1-i}, \vec{x}^{i}, \vec{s}^{n-j}, \vec{y}^{j}\right) \\
& \times \bar{f}_{v}\left(s_{n-j+1}, \ldots, s_{l^{\prime}-j-i},{\overrightarrow{x^{\prime}}}^{i}, t_{m-i}, \ldots, t_{l^{\prime}-i-j},{\overrightarrow{y^{\prime}}}^{j}\right) \phi\left(y_{1}, y_{1}^{\prime}\right) \ldots \phi\left(y_{j}, y_{j}^{\prime}\right) \mathrm{d} \vec{y}^{j} \mathrm{~d}{\overrightarrow{y^{\prime}}}^{j}
\end{aligned}
$$


Then we obtain that

$$
\frac{1}{m^{2}}\left\|D .\left(I_{m, n}(f)\right)\right\|_{\mathfrak{H}}^{2}=\sum_{r=0}^{l^{\prime}} \int_{[0, \infty)^{2}} \mathrm{~d} u \mathrm{~d} v \phi(u, v) I_{l^{\prime}-r, l^{\prime}-r}\left(g_{r}(u, v)\right) .
$$

where

$$
g_{k}(u, v)=\sum_{i+j=k}\left(\begin{array}{c}
m-1 \\
i
\end{array}\right)^{2}\left(\begin{array}{c}
n \\
j
\end{array}\right)^{2} i ! j ! f_{u}\left(\vec{t}^{m-1}, \vec{s}^{n}\right) \tilde{\otimes}_{i, j} h^{v}\left(\vec{t}^{n}, \vec{s}^{m-1}\right) .
$$

Taking expectation to Eq.(2.27), we have that

$$
\begin{aligned}
\frac{1}{m^{2}} \mathbb{E}\left[\left\|D \cdot\left(I_{m, n}(f)\right)\right\|_{\mathfrak{H}}^{2}\right] & =\int_{[0, \infty)^{2}} \mathrm{~d} u \mathrm{~d} v \phi(u, v) g_{l^{\prime}}(u, v) \\
& =(m-1) ! n ! \int_{[0, \infty)^{2}} \mathrm{~d} u \mathrm{~d} v \phi(u, v) f_{u} \otimes_{m-1, n} h^{v} \\
& =(m-1) ! n !\|f\|_{\mathfrak{H}^{\otimes(m+n)}}^{2} .
\end{aligned}
$$

Step 2: Calculating variance. It follows from Fubini's theorem and Itô's isometry that we have:

$$
\begin{aligned}
& \frac{1}{m^{4}} \mathbb{E}\left[\left\|D .\left(I_{m, n}(f)\right)\right\|_{\mathfrak{H}}^{4}\right] \\
& =\sum_{r=0}^{l^{\prime}} \int_{[0, \infty)^{4}} \mathrm{~d} u \mathrm{~d} v \mathrm{~d} u^{\prime} \mathrm{d} v^{\prime} \phi(u, v) \phi\left(u^{\prime}, v^{\prime}\right)\left[\left(l^{\prime}-r\right) !\right]^{2}\left\langle g_{r}(u, v), g_{r}\left(u^{\prime}, v^{\prime}\right)\right\rangle_{\mathfrak{H}^{\otimes 2\left(l^{\prime}-r\right)}} .
\end{aligned}
$$

It is easy to check that

$$
\begin{aligned}
& \int_{[0, \infty)^{4}} \mathrm{~d} u \mathrm{~d} v \mathrm{~d} u^{\prime} \mathrm{d} v^{\prime} \phi(u, v) \phi\left(u^{\prime}, v^{\prime}\right)\left\langle f_{u} \tilde{\otimes}_{i, j} h^{v}, f_{u^{\prime}} \tilde{\otimes}_{i, j} h^{v^{\prime}}\right\rangle_{\mathfrak{H}^{\otimes 2\left(l^{\prime}-k\right)}} \\
& =\left\langle f \tilde{\otimes}_{i+1, j} h, f \tilde{\otimes}_{i+1, j} h\right\rangle_{\mathfrak{H} \otimes 2\left(l^{\prime}-k\right)}=\left\|f \tilde{\otimes}_{i+1, j} h\right\|_{\mathfrak{H}^{\otimes 2\left(l^{\prime}-k\right)}}^{2},
\end{aligned}
$$

which implies that

$$
\frac{1}{m^{4}} \mathbb{E}\left[\left\|D .\left(I_{m, n}(f)\right)\right\|_{\mathfrak{H}}^{4}\right]=\sum_{r=0}^{l^{\prime}}\left[\left(l^{\prime}-r\right) !\right]^{2}\left\langle G_{r}, G_{r}\right\rangle_{\mathfrak{H} \otimes 2\left(l^{\prime}-r\right)},
$$

where

$$
G_{k}=\sum_{i+j=k}\left(\begin{array}{c}
m-1 \\
i
\end{array}\right)^{2}\left(\begin{array}{c}
n \\
j
\end{array}\right)^{2} i ! j ! f\left(\vec{t}^{m}, \vec{s}^{n}\right) \tilde{\otimes}_{i+1, j} h\left(\vec{t}^{n}, \vec{s}^{m}\right) .
$$

Especially, for the term with $k=l^{\prime}$, we have that

$$
\begin{aligned}
\left|G_{l^{\prime}}\right|^{2} & =[(m-1) ! n !]^{2}\left|f \otimes_{m, n} h\right|^{2} \\
& =[(m-1) ! n !]^{2}\|f\|_{\mathfrak{H} \otimes(m+n)}^{4}=\left(\frac{1}{m^{2}} \mathbb{E}\left[\left\|D .\left(I_{m, n}(f)\right)\right\|_{\mathfrak{H}}^{2}\right]\right)^{2} .
\end{aligned}
$$

Substituting the above equality displayed into (2.30), we have that

$$
\begin{aligned}
\operatorname{Var}\left(\left\|D I_{m, n}(f)\right\|_{\mathfrak{H}}^{2}\right) & =m^{4} \sum_{r^{\prime}=0}^{l^{\prime}-1}\left[\left(l^{\prime}-r^{\prime}\right) !\right]^{2}\left\langle G_{r^{\prime}}, G_{r^{\prime}}\right\rangle_{\mathfrak{H} \otimes 2\left(l^{\prime}-r^{\prime}\right)} \\
& =\sum_{r=1}^{l-1}[(l-r) !]^{2}\left\|\eta_{r}\right\|_{\mathfrak{H} \otimes(2(l-r))}^{2} \quad\left(\text { let } l=l^{\prime}+1, r=r^{\prime}+1\right),
\end{aligned}
$$


where $\eta_{r}=m^{2} G_{r^{\prime}}$, which implies the desired expressions (2.20) and (2.23).

Proof of Theorem 1.3. Since (i) $\Rightarrow$ (ii) is well known, we need only to show the following implications:

$$
\text { (ii) } \Rightarrow \text { (iii) } \Rightarrow \text { (iv) } \Rightarrow \text { (v) } \Rightarrow \text { (i) }
$$

$[($ ii) $\Rightarrow$ (iii) ] Condition (ii) implies that as $k \rightarrow \infty$,

$$
\mathbb{E}\left[\left|F_{k}\right|^{4}\right]-2\left(\mathbb{E}\left[\left|F_{k}\right|^{2}\right]\right)^{2}-\left|\mathbb{E}\left[F_{k}^{2}\right]\right|^{2} \rightarrow 0,
$$

which implies that Condition (iii) holds by (2.8)-(2.9), (see Lemma 2.3).

$[($ iii $) \Rightarrow($ iv)] The inequality (5.2) of Itô (1952) implies that when Condition (iii) holds, we have that as $k \rightarrow \infty$,

$\left\|f_{k} \tilde{\otimes}_{i, j} f_{k}\right\|_{\mathfrak{H} \otimes(2(l-i-j))} \rightarrow 0, \quad\left\|f_{k} \tilde{\otimes}_{i, j} h_{k}\right\|_{\mathfrak{H} \otimes(2(l-i-j))}=\left\|h_{k} \tilde{\otimes}_{i, j} f_{k}\right\|_{\mathfrak{H} \otimes(2(l-i-j))} \rightarrow 0$.

$[(\mathrm{iv}) \Rightarrow(\mathrm{v})]$ It follows from Minkowski's inequality and Proposition 2.6 that as $k \rightarrow \infty$,

$$
\eta_{r}^{k} \rightarrow 0, \quad \xi_{r}^{k} \rightarrow 0, \quad \nu_{r}^{k} \rightarrow 0, \quad r=1, \ldots, l-1,
$$

where $\eta_{r}^{k}, \xi_{r}^{k}, \nu_{r}^{k}$ are given as Equations (2.20)-(2.22). By (2.23)-(2.25), we obtain that Condition (iv) holds.

$[(\mathrm{v}) \Rightarrow(\mathrm{i})]$ We follow the idea of Nualart and Ortiz-Latorre (2008, Theorem 4), i.e. we combine Malliavin calculus and partial differential equations. Let

$$
\varphi_{k}(z)=\mathbb{E}\left[e^{\mathrm{i}\left(\bar{z} F_{k}+z \bar{F}_{k}\right) / 2}\right] .
$$

Then we have that

$$
\left\{\begin{array}{l}
\frac{\partial \varphi_{k}}{\partial z}=\frac{\mathrm{i}}{2} \mathbb{E}\left[\bar{F}_{k} \times e^{\mathrm{i}\left(\bar{z} F_{k}+z \bar{F}_{k}\right) / 2}\right] \\
\frac{\partial \varphi_{k}}{\partial \bar{z}}=\frac{\mathrm{i}}{2} \mathbb{E}\left[F_{k} \times e^{\mathrm{i}\left(\bar{z} F_{k}+z \bar{F}_{k}\right) / 2}\right] .
\end{array}\right.
$$

By the assumption $\mathbb{E}\left[\left|F_{k}\right|^{2}\right] \rightarrow \sigma^{2},\left\{F_{k}\right\}$ are tight. Now suppose that the subsequence $\left\{F_{n_{k}}\right\}$ converges to $G$ in law. Without ambiguity, we still denote $\left\{F_{n_{k}}\right\}$ by $\left\{F_{k}\right\}$. By the hypercontractivity inequality of complex multiple Wiener-Itô integrals, see e.g. Chen (2014), $\left\{\left|F_{k}\right|^{r}\right\}$ is uniformly integrable and thus $\mathbb{E}\left[|G|^{r}\right]=$ $\lim _{k \rightarrow \infty} \mathbb{E}\left[\left|F_{k}\right|^{r}\right]$ for all $r \geq 1$, see e.g. Billingsley (1968, Theorem 5.4). Therefore, the characteristic function $\varphi(z)=\mathbb{E}\left[e^{\frac{i}{2}(\bar{z} G+z \bar{G})}\right]$ has continuous partial derivatives of any order.

It is not difficult to see that

$$
\begin{aligned}
\mathbb{E}\left[\bar{F}_{k} \times e^{\mathrm{i}\left(\bar{z} F_{k}+z \bar{F}_{k}\right) / 2}\right] & =\frac{1}{m} \mathbb{E}\left[\overline{(\delta D) F_{k}} \times e^{\mathrm{i}\left(\bar{z} F_{k}+z \bar{F}_{k}\right) / 2}\right] \\
& =\frac{1}{m} \mathbb{E}\left[\left\langle D\left(e^{\mathrm{i}\left(\bar{z} F_{k}+z \bar{F}_{k}\right) / 2}\right), D F_{k}\right\rangle_{\mathfrak{H}}\right] \\
& =\frac{1}{m} \mathbb{E}\left[\left\langle e^{\mathrm{i}\left(\bar{z} F_{k}+z \bar{F}_{k}\right) / 2}\left(\bar{z} D F_{k}+z D \bar{F}_{k}\right), D F_{k}\right\rangle_{\mathfrak{H}}\right]
\end{aligned}
$$

Clearly, for any $z \in \mathbb{C}, e^{\mathrm{i}\left(\bar{z} F_{k}+z \bar{F}_{k}\right) / 2} \rightarrow e^{\mathrm{i}(\bar{z} G+z \bar{G}) / 2}$ in $L^{2}(\Omega)$. Thus, Condition (iv) implies that as $k \rightarrow \infty$,

$$
\begin{aligned}
& \mathbb{E}\left[\left\langle e^{\mathrm{i}\left(\bar{z} F_{k}+z \bar{F}_{k}\right) / 2}\left(\bar{z} D F_{k}+z D \bar{F}_{k}\right), D F_{k}\right\rangle_{\mathfrak{H}}\right] \\
& \rightarrow\left(\bar{z} \lim _{k \rightarrow \infty} \mathbb{E}\left[\left\|D F_{k}\right\|_{\mathfrak{H}}^{2}\right]+z \lim _{k \rightarrow \infty} \mathbb{E}\left[\left\langle D \bar{F}_{k} D F_{k}\right\rangle_{\mathfrak{H}}\right]\right) \varphi(z), \quad \forall z \in \mathbb{C},
\end{aligned}
$$


since the scalar product in the Hilbert space $L^{2}(\Omega)$ depends continuously on its factors. It follows from (2.29) and

$$
\frac{1}{m n} \mathbb{E}\left[\left\langle D I_{m, n}(f), D \overline{I_{m, n}(f)}\right\rangle_{\mathfrak{H}}\right]=\delta_{m, n} m !(m-1) ! f \otimes_{m, m} f
$$

that

$$
\begin{aligned}
\lim _{k \rightarrow \infty} \mathbb{E}\left[\left\|D F_{k}\right\|_{\mathfrak{H}}^{2}\right] & =m \lim _{k \rightarrow \infty} \mathbb{E}\left[\left\|F_{k}\right\|_{\mathfrak{H}}^{2}\right]=m \sigma^{2}, \\
\lim _{k \rightarrow \infty} \mathbb{E}\left[\left\langle D \bar{F}_{k} D F_{k}\right\rangle_{\mathfrak{H}}\right] & =m \delta_{m, n} \lim _{k \rightarrow \infty} \overline{\mathbb{E}\left[F_{k}^{2}\right]} \\
& =m(c-\mathrm{i} b) \delta_{m, n} .
\end{aligned}
$$

Therefore, it follows from (2.33) that for any $z \in \mathbb{C}$,

$$
\frac{\partial \varphi}{\partial z}=\left[\bar{z} \sigma^{2}+z \cdot(c-\mathrm{i} b) \delta_{m, n}\right] \varphi(z) .
$$

In the same way,

$$
\frac{\partial \varphi}{\partial \bar{z}}=\left[\bar{z} \cdot(c+\mathrm{i} b) \delta_{m, n}+z \sigma^{2}\right] \varphi(z) .
$$

Clearly, $\varphi(0)=1$. Therefore, $G$ is a bivariate normal distribution with covariance matrix $\mathrm{C}=\frac{1}{2}\left[\begin{array}{cc}\sigma^{2}+c & b \\ b & \sigma^{2}-c\end{array}\right]$. Prokhorov's theorem implies that $\left\{F_{k}\right\}$ converges to a bivariate normal distribution with the desired covariance matrix $\mathrm{C}$.

\section{Asymptotic consistency and normality}

We need several propositions and lemmas before the proof of Theorem 1.1. The following lemma's proof is easy.

Lemma 3.1. For any $H \in\left(\frac{1}{2}, 1\right)$, we have that

$$
\int_{[0, \infty)^{2}} e^{-\gamma u_{1}-\bar{\gamma} u_{2}}\left|u_{1}-u_{2}\right|^{2 H-2} \mathrm{~d} u_{1} \mathrm{~d} u_{2}=d
$$

where d is defined by (1.7).

Proposition 3.2. Let $Z$ be the solution to (1.1). As $T \rightarrow \infty$, we have that

$$
\frac{1}{T} \int_{0}^{T}\left|Z_{t}\right|^{2} \mathrm{~d} t \rightarrow a \alpha_{H} d, \quad \text { a.s. }
$$

Proof: Denote $Y_{t}=\sqrt{a} \int_{-\infty}^{t} e^{-\gamma(t-s)} \mathrm{d} \zeta_{s}$. It is easy to see that $Y$ is centered complex Gaussian process. Itô's isometry implies that for any $t \in \mathbb{R}, s \geq 0$,

$$
\mathbb{E}\left[Y_{t+s} \bar{Y}_{t}\right]=\alpha_{H} a \int_{-s}^{\infty} \mathrm{d} v_{1} \int_{0}^{\infty} \mathrm{d} v_{2} e^{-\gamma\left(v_{1}+s\right)} e^{-\bar{\gamma} v_{2}}\left|v_{1}-v_{2}\right|^{2 H-2}=\mathbb{E}\left[Y_{s} \bar{Y}_{0}\right] .
$$

Thus $Y_{t}$ is stationary. It is easy to check that as $s \rightarrow \infty, \mathbb{E}\left[Y_{s} \bar{Y}_{0}\right] \rightarrow 0$ with the same order as $|s|^{2 H-2}$, which implies that $\left\{Y_{t}\right\}$ is ergodic, see e.g. Dym and McKean (1976, p. 78). 
Then we have that

$$
\begin{aligned}
Z_{t} & =e^{-\gamma t} Z_{0}+\sqrt{a} \int_{0}^{t} e^{-\gamma(t-s)} \mathrm{d} \zeta_{s} \\
& =e^{-\gamma t} Z_{0}+\sqrt{a} \int_{-\infty}^{t} e^{-\gamma(t-s)} \mathrm{d} \zeta_{s}-e^{-\gamma t} \sqrt{a} \int_{-\infty}^{0} e^{\gamma s} \mathrm{~d} \zeta_{s} \\
& =Y_{t}+e^{-\gamma t}\left(Z_{0}-Y_{0}\right) .
\end{aligned}
$$

The ergodicity and Cauchy-Schwarz inequality imply that as $T \rightarrow \infty$,

$$
\begin{aligned}
\frac{1}{T} \int_{0}^{T}\left|Z_{t}\right|^{2} \mathrm{~d} t & =\frac{1}{T} \int_{0}^{T}\left[\left|Y_{t}\right|^{2}-2 \Re\left(e^{-\gamma t}\left(Z_{0}-Y_{0}\right) \bar{Y}_{t}\right)+e^{-2 \lambda t}\left|Z_{0}-Y_{0}\right|^{2}\right] \mathrm{d} t \\
& \rightarrow \lim _{T \rightarrow \infty} \mathbb{E}\left[\left|Y_{T}\right|^{2}\right]=a \alpha_{H} d, \quad \text { a.s. }
\end{aligned}
$$

where the last equality is from Eq. (3.3) and Lemma 3.1.

Denote

$$
\psi_{t}(r, s)=e^{-\bar{\gamma}(r-s)} \mathbf{1}_{\{0 \leq s \leq r \leq t\}} \quad \text { and } \quad X_{t}=I_{1,1}\left(\psi_{t}(r, s)\right) .
$$

Lemma 3.3. As $n \rightarrow \infty$, the sequence $\left\{\xi_{n}:=\frac{1}{n} X_{n}\right\}$ converges to zero almost surely.

Proof: Denote $F_{T}=\frac{1}{\sqrt{T}} X_{T}$. Lemma 3.5 implies that $\sup _{n} \mathbb{E}\left[\left|F_{n}\right|^{2}\right]<\infty$. From the hypercontractivity of multiple Wiener-Itô integrals, we see that $\sup _{n} \mathbb{E}\left[\left|F_{n}\right|^{4}\right]<\infty$. For any fixed $\varepsilon>0$, it follows from Chebyshev's inequality that

$$
P\left(\left|\xi_{n}\right|>\varepsilon\right)=P\left(\left|F_{n}\right|>\sqrt{n} \varepsilon\right) \leq \frac{1}{n^{2} \varepsilon^{4}} \mathbb{E}\left[\left|F_{n}\right|^{4}\right] \leq \frac{3^{4}}{n^{2} \varepsilon^{4}} \mathbb{E}\left[\left|F_{n}\right|^{2}\right]^{2} .
$$

The Borel-Cantelli lemma implies that $\left\{\xi_{n}\right\}$ converges to zero almost surely.

Proposition 3.4. For any real number $p \geq 2$ and integer $n \geq 1$,

$$
B_{n}:=\int_{n}^{n+1} \int_{n}^{n+1} \frac{\left|X_{t}-X_{s}\right|^{p}}{|t-s|^{2 p H}} \mathrm{~d} s \mathrm{~d} t
$$

is finite. Moreover, for any real numbers $p>2, q>1$ and integer $n \geq 1$,

$$
\left|X_{t_{2}}-X_{t_{1}}\right| \leq R_{p, q} n^{q / p}, \quad \forall t_{1}, t_{2} \in[n, n+1],
$$

where $R_{p, q}$ is a random constant independent of $n$.

Proof: For any $n \leq t_{1} \leq t_{2} \leq n+1$, Itô's isometry implies that

$$
\begin{aligned}
& \mathbb{E}\left[\left|X_{t_{2}}-X_{t_{1}}\right|^{2}\right]=\left\|\psi_{t_{2}}(r, s)-\psi_{t_{1}}(r, s)\right\|_{\mathfrak{H}^{\otimes 2}}^{2}=\left\|e^{-\bar{\gamma}(r-s)} \mathbf{1}_{\left\{t_{1}<s \leq r \leq t_{2}\right\}}\right\|_{\mathfrak{H}^{\otimes 2}}^{2} \\
& =\int_{t_{1}<s_{1} \leq r_{1} \leq t_{2}} \int_{t_{1}<s_{2} \leq r_{2} \leq t_{2}} e^{-\bar{\gamma}\left(r_{1}-s_{1}\right)-\gamma\left(r_{2}-s_{2}\right)} \phi\left(r_{1}, r_{2}\right) \phi\left(s_{1}, s_{2}\right) \mathrm{d} s_{1} \mathrm{~d} s_{2} \mathrm{~d} r_{1} \mathrm{~d} r_{2} \\
& \leq \int_{t_{1}<s_{1} \leq r_{1} \leq t_{2}} \int_{t_{1}<s_{2} \leq r_{2} \leq t_{2}} \phi\left(r_{1}, r_{2}\right) \phi\left(s_{1}, s_{2}\right) \mathrm{d} s_{1} \mathrm{~d} s_{2} \mathrm{~d} r_{1} \mathrm{~d} r_{2}=\left(t_{2}-t_{1}\right)^{4 H} .
\end{aligned}
$$

The hypercontractivity of multiple Wiener-Itô integrals implies that for any $p \geq 2$ and any $n \leq t_{1} \leq t_{2} \leq n+1$,

$$
\begin{aligned}
\mathbb{E}\left[\left|X_{t_{2}}-X_{t_{1}}\right|^{p}\right] & \leq(p-1)^{p} \mathbb{E}\left[\left|X_{t_{2}}-X_{t_{1}}\right|^{2}\right]^{\frac{p}{2}} \\
& \leq(p-1)^{p}\left(t_{2}-t_{1}\right)^{2 p H} .
\end{aligned}
$$


Take $\Psi(x)=x^{p}$ and $\rho(x)=x^{2 H}$. The above inequality yields

$$
\mathbb{E}\left(B_{n}\right)=\mathbb{E}\left[\int_{n}^{n+1} \int_{n}^{n+1} \Psi\left(\frac{\left|X_{t}-X_{s}\right|}{\rho(|t-s|)}\right) \mathrm{d} s \mathrm{~d} t\right] \leq(p-1)^{p} .
$$

For any $q>1$, we have

$$
\mathbb{E}\left(\sum_{n=1}^{\infty} \frac{B_{n}}{n^{q}}\right)=\sum_{n=1}^{\infty} \frac{\mathbb{E}\left(B_{n}\right)}{n^{q}}<\infty
$$

This implies that

$$
\sum_{n=1}^{\infty} \frac{B_{n}}{n^{q}} \leq R_{p, q} \quad \text { for some random constant } R_{p, q} .
$$

Or we have

$$
B_{n} \leq R_{p, q} n^{q} \quad \text { for all positive number } q>1 \text { and integer } n \geq 1 .
$$

An application of the Garsia-Rodemich-Rumsey inequality, see e.g. Hu (2017, Theorem 2.1)), implies that

$$
\left|X_{t}-X_{s}\right| \leq 8 \int_{0}^{|t-s|} \Psi^{-1}\left(\frac{4 B_{n}}{u^{2}}\right) \rho^{\prime}(u) \mathrm{d} u=16 H \frac{\left(4 B_{n}\right)^{1 / p}}{2 H-\frac{2}{p}}|t-s|^{2 H-\frac{2}{p}} \leq c_{p} B_{n}^{\frac{1}{p}} .
$$

This combined with (3.8) proves the proposition.

Denote

$$
h_{t}(r, s)=e^{-\gamma(-r+s)} \mathbf{1}_{\{0 \leq r \leq s \leq t\}} .
$$

Lemma 3.5. Let $H \in\left(\frac{1}{2}, \frac{3}{4}\right)$. Then the following integrals are absolutely convergent

$$
\begin{aligned}
& \lim _{T \rightarrow \infty} \frac{1}{\alpha_{H}^{2} T} \int_{[0, T]^{4}} \psi_{T}\left(t_{1}, s_{1}\right) \overline{\psi_{T}\left(t_{2}, s_{2}\right)} \phi\left(t_{1}, t_{2}\right) \phi\left(s_{1}, s_{2}\right) \mathrm{d} t_{1} \mathrm{~d} t_{2} \mathrm{~d} s_{1} \mathrm{~d} s_{2}=\sigma^{2} \\
& \lim _{T \rightarrow \infty} \frac{1}{\alpha_{H}^{2} T} \int_{[0, T]^{4}} \psi_{T}\left(t_{1}, s_{1}\right) \overline{h_{T}\left(t_{2}, s_{2}\right)} \phi\left(t_{1}, t_{2}\right) \phi\left(s_{1}, s_{2}\right) \mathrm{d} t_{1} \mathrm{~d} t_{2} \mathrm{~d} s_{1} \mathrm{~d} s_{2}=c+\mathrm{i} b,
\end{aligned}
$$

where $\sigma^{2}$ and $c, b$ are defined by (1.5) and (1.6).

Proof: We only evaluate the first integral since the other one is similar. We divide the domain $\left\{0 \leq s_{1} \leq t_{1} \leq T, 0 \leq s_{2} \leq t_{2} \leq T\right\}$ into six disjoint regions according to the distinct orders of $s_{1}, t_{1}, s_{2}, t_{2}$ :

$$
\begin{array}{ll}
\Delta_{1}=\left\{0 \leq s_{2} \leq t_{2} \leq s_{1} \leq t_{1} \leq T\right\}, & \Delta_{2}=\left\{0 \leq s_{1} \leq t_{1} \leq s_{2} \leq t_{2} \leq T\right\} \\
\Delta_{3}=\left\{0 \leq s_{1} \leq s_{2} \leq t_{1} \leq t_{2} \leq T\right\}, & \Delta_{4}=\left\{0 \leq s_{2} \leq s_{1} \leq t_{2} \leq t_{1} \leq T\right\} \\
\Delta_{5}=\left\{0 \leq s_{1} \leq s_{2} \leq t_{2} \leq t_{1} \leq T\right\}, & \Delta_{6}=\left\{0 \leq s_{2} \leq s_{1} \leq t_{1} \leq t_{2} \leq T\right\}
\end{array}
$$

We also denote $I_{i}=\frac{1}{T} \int_{\Delta_{i}} \psi_{T}\left(t_{1}, s_{1}\right) \overline{\psi_{T}\left(t_{2}, s_{2}\right)} \phi\left(t_{1}, t_{2}\right) \phi\left(s_{1}, s_{2}\right) \mathrm{d} t_{1} \mathrm{~d} t_{2} \mathrm{~d} s_{1} \mathrm{~d} s_{2}, i=$ $1, \cdots, 6$.

Firstly, we consider $I_{1}$. It follows from L'Hospital rule that

$$
\lim _{T \rightarrow \infty} I_{1}=\lim _{T \rightarrow \infty} \int_{0}^{T} \mathrm{~d} s_{1} \int_{0}^{s_{1}} \mathrm{~d} t_{2} \int_{0}^{t_{2}} \mathrm{~d} s_{2} e^{-\bar{\gamma}\left(T-s_{1}\right)} e^{-\gamma\left(t_{2}-s_{2}\right)} \phi\left(T, t_{2}\right) \phi\left(s_{1}, s_{2}\right) .
$$


Making substitution $a=t_{2}-s_{2}, b=s_{1}-t_{2}, c=T-s_{1}$, we have that as $T \rightarrow \infty$

$$
\begin{aligned}
& \lim _{T \rightarrow \infty} I_{1}=\alpha_{H}^{2} \lim _{T \rightarrow \infty} \int_{a, b, c \geq 0, a+b+c \leq T} \mathrm{~d} a \mathrm{~d} b \mathrm{~d} c e^{-\bar{\gamma} c} e^{-\gamma a}[(b+c)(a+b)]^{2 H-2} \\
& =\alpha_{H}^{2} \int_{[0, \infty)^{3}} \mathrm{~d} a \mathrm{~d} b \mathrm{~d} c e^{-\bar{\gamma} c} e^{-\gamma a}[(b+c)(a+b)]^{2 H-2} .
\end{aligned}
$$

The above integral is absolutely convergent when $H \in\left(\frac{1}{2}, \frac{3}{4}\right)$. In fact, since

$$
(b+c)(a+b) \geq a c \mathbf{1}_{[0,1]}(b)+b^{2} \mathbf{1}_{[1, \infty)}(b),
$$

we have that

$$
\begin{aligned}
& \left|\int_{[0, \infty)^{3}} \mathrm{~d} a \mathrm{~d} b \mathrm{~d} c e^{-\bar{\gamma} c} e^{-\gamma a}[(b+c)(a+b)]^{2 H-2}\right| \\
& \leq \int_{[0, \infty)^{3}} \mathrm{~d} a \mathrm{~d} b \mathrm{~d} c e^{-\lambda c} e^{-\lambda a}\left[b^{4 H-4} \mathbf{1}_{[1, \infty)}+(a c)^{2 H-2} \mathbf{1}_{[0,1]}(b)\right] \\
& =\frac{1}{(3-4 H) \lambda^{2}}+\left(\frac{\Gamma(2 H-1)}{\lambda^{2 H-1}}\right)^{2} .
\end{aligned}
$$

Substituting the equality of Gamma function $\int_{0}^{\infty} e^{-x \beta} x^{\alpha-1}=\frac{\Gamma(\alpha)}{\beta^{\alpha}}$ with $\alpha>$ $0, \Re \beta>0$ into (3.11), we have that

$$
\begin{aligned}
& \int_{[0, \infty)^{3}} \mathrm{~d} a \mathrm{~d} b \mathrm{~d} c e^{-\bar{\gamma} c} e^{-\gamma a}[(b+c)(a+b)]^{2 H-2} \\
& =\frac{1}{\Gamma(2-2 H)^{2}} \int_{[0, \infty)^{5}} \mathrm{~d} a \mathrm{~d} b \mathrm{~d} c \mathrm{~d} x \mathrm{~d} y e^{-\bar{\gamma} c} e^{-\gamma a} e^{-x(b+c)} x^{1-2 H} e^{-y(a+b)} y^{1-2 H} \\
& =\frac{1}{\Gamma(2-2 H)^{2}} \int_{[0, \infty)^{2}} \mathrm{~d} x \mathrm{~d} y \frac{(x y)^{1-2 H}}{(x+y)(x+\bar{\gamma})(y+\gamma)}
\end{aligned}
$$

It is easy to see that $I_{2}=I_{1}$. In a similar way as for $I_{1}$, we have

$$
\begin{aligned}
\lim _{T \rightarrow \infty} I_{3} & =\lim _{T \rightarrow \infty} I_{4}=\frac{\alpha_{H}^{2} \Gamma^{2}(2 H-1)}{2 \lambda|\gamma|^{4 H-2}}, \\
\lim _{T \rightarrow \infty} I_{5} & =\frac{\alpha_{H}^{2} \Gamma^{2}(2 H-1)}{2 \lambda \bar{\gamma}^{4 H-2}}, \\
\lim _{T \rightarrow \infty} I_{6} & =\frac{\alpha_{H}^{2} \Gamma^{2}(2 H-1)}{2 \lambda \gamma^{4 H-2}} .
\end{aligned}
$$

Finally, by adding (3.12)-(3.15) together, we get (1.5).

Lemma 3.6. Let $\psi_{T}, h_{T}$ be as in (3.4) and (3.9) respectively. As $T \rightarrow \infty$, we have that:

$$
\frac{1}{T} \psi_{T} \otimes_{0,1} \psi_{T} \rightarrow 0, \frac{1}{T} \psi_{T} \otimes_{1,0} \psi_{T} \rightarrow 0, \frac{1}{T} \psi_{T} \otimes_{0,1} h_{T} \rightarrow 0, \frac{1}{T} \psi_{T} \otimes_{1,0} h_{T} \rightarrow 0, \text { in } \mathfrak{H}^{\otimes 2}
$$


Proof: When $0<t<s<T$, we have that

$$
\begin{aligned}
& \frac{1}{\alpha_{H}}\left|\psi_{T} \otimes_{0,1} \psi_{T}(t, s)\right|=\left|\int_{0}^{t} \mathrm{~d} u_{1} \int_{s}^{T} \mathrm{~d} u_{2} e^{-\bar{\gamma}\left(t-u_{1}\right)} e^{-\bar{\gamma}\left(u_{2}-s\right)}\right| u_{1}-\left.u_{2}\right|^{2 H-2} \mid \\
\leq & \int_{0}^{t} \mathrm{~d} u_{1} \int_{s}^{T} \mathrm{~d} u_{2} e^{-\lambda\left(t-u_{1}\right)} e^{-\lambda\left(u_{2}-s\right)}\left|u_{2}-u_{1}\right|^{2 H-2} \\
\leq & (s-t)^{2 H-2} \int_{0}^{t} \mathrm{~d} u_{1} \int_{s}^{T} \mathrm{~d} u_{2} e^{-\lambda\left(t-u_{1}\right)} e^{-\lambda\left(u_{2}-s\right)} \leq \frac{1}{\lambda^{2}}(s-t)^{2 H-2}
\end{aligned}
$$

When $s<t$, we have that

$$
\begin{aligned}
& \int_{0}^{t} \mathrm{~d} u_{1} \int_{s}^{T} \mathrm{~d} u_{2} e^{-\lambda\left(t-u_{1}\right)} e^{-\lambda\left(u_{2}-s\right)}\left|u_{2}-u_{1}\right|^{2 H-2} \\
&=\left(\int_{0}^{s} \mathrm{~d} u_{1} \int_{s}^{T} \mathrm{~d} u_{2}+\int_{s}^{t} \mathrm{~d} u_{1} \int_{s}^{t} \mathrm{~d} u_{2}+\int_{s}^{t} \mathrm{~d} u_{1} \int_{t}^{T} \mathrm{~d} u_{2}\right) e^{-\lambda\left(t-u_{1}\right)} \\
& e^{-\lambda\left(u_{2}-s\right)}\left|u_{2}-u_{1}\right|^{2 H-2}=I_{1}(T)+I_{2}(T)+I_{3}(T) .
\end{aligned}
$$

For the first term, we have that

$$
\begin{aligned}
I_{1}(T) & =e^{-\lambda(t-s)} \int_{0}^{s} \mathrm{~d} u_{1} \int_{s-u_{1}}^{T-u_{1}} \mathrm{~d} z e^{-\lambda z} z^{2 H-2} \\
& =e^{-\lambda(t-s)} \int_{0}^{T} \mathrm{~d} z e^{-\lambda z} z^{2 H-2} \int_{0 \vee(s-z)}^{s \wedge(T-z)} \mathrm{d} u_{1} \\
& \leq e^{-\lambda(t-s)} \int_{0}^{T} \mathrm{~d} z e^{-\lambda z} z^{2 H-2}[s-(s-z)] \\
& \leq e^{-\lambda(t-s)} \frac{\Gamma(2 H)}{\lambda^{2 H}} \leq c_{\lambda, H}|t-s|^{2 H-2},
\end{aligned}
$$

where $c_{\lambda, H}$ is a constant independent of $T$. For the second term, we have that

$$
\begin{aligned}
I_{2}(T) & =2 e^{-\lambda(t-s)} \int_{s}^{t} \mathrm{~d} u_{1} \int_{s}^{u_{1}} \mathrm{~d} u_{2} e^{\lambda\left(u_{1}-u_{2}\right)}\left(u_{1}-u_{2}\right)^{2 H-2} \\
& =2 e^{-\lambda(t-s)} \int_{s}^{t} \mathrm{~d} u_{1} \int_{0}^{u_{1}-s} \mathrm{~d} z e^{\lambda z} z^{2 H-2} \\
& =2 e^{-\lambda(t-s)} \int_{0}^{t-s} \mathrm{~d} z e^{\lambda z} z^{2 H-2}(t-s-z) \leq c_{\lambda, H}|t-s|^{2 H-2},
\end{aligned}
$$

where $c_{\lambda, H}$ is a constant independent of $T$ and the last inequality is by means of L'Hospital rule. In fact, when $H \in\left(\frac{1}{2}, 1\right)$, then we have that

$$
\lim _{x \rightarrow \infty} \frac{\int_{0}^{x} \mathrm{~d} z e^{\lambda z} z^{2 H-2}(x-z)}{e^{\lambda x} x^{2 H-2}}=\frac{1}{\lambda^{2}} .
$$


For the third term, we have that

$$
\begin{aligned}
I_{3}(T) & =e^{-\lambda(t-s)} \int_{s}^{t} \mathrm{~d} u_{1} \int_{t-u_{1}}^{T-u_{1}} \mathrm{~d} z e^{-\lambda z} z^{2 H-2} \\
& =e^{-\lambda(t-s)} \int_{0}^{T} \mathrm{~d} z e^{-\lambda z} z^{2 H-2} \int_{s \vee(t-z)}^{t \wedge(T-z)} \mathrm{d} u_{1} \\
& \leq e^{-\lambda(t-s)} \int_{0}^{t-s} \mathrm{~d} z e^{-\lambda z} z^{2 H-2}[t-(t-z)] \\
& \leq e^{-\lambda(t-s)} \frac{\Gamma(2 H)}{\lambda^{2 H}} \leq c_{\lambda, H}|t-s|^{2 H-2},
\end{aligned}
$$

where $c_{\lambda, H}$ is a constant independent of $T$. Thus, we have that

$$
\left|\int_{0}^{t} \mathrm{~d} u_{1} \int_{s}^{T} \mathrm{~d} u_{2} e^{-\bar{\gamma}\left(t-u_{1}\right)} e^{-\bar{\gamma}\left(u_{2}-s\right)}\right| u_{1}-\left.u_{2}\right|^{2 H-2}\left|\leq c_{\lambda, H}\right| t-\left.s\right|^{2 H-2} .
$$

This inequality together with the inequality (3.17) implies that

$$
\begin{aligned}
\left\|\frac{1}{T} \psi_{T} \otimes_{0,1} \psi_{T}\right\|_{\mathfrak{H}^{\otimes 2}}^{2} & \leq \frac{\alpha_{H}^{2} c_{\lambda, H}^{2}}{T^{2}}\|\phi\|_{\mathfrak{H} \otimes 2}^{2} \\
& =\frac{\alpha_{H}^{2} c_{\lambda, H}^{2}}{T^{2}} \int_{[0, T]^{4}} \mathrm{~d} t_{1} \mathrm{~d} t_{2} \mathrm{~d} s_{1} \mathrm{~d} s_{2} \phi\left(t_{1}, s_{1}\right) \phi\left(t_{2}, s_{2}\right) \phi\left(t_{1}, t_{2}\right) \phi\left(s_{1}, s_{2}\right) .
\end{aligned}
$$

As $T \rightarrow \infty$, L'Hospital rule and the symmetric property of the above integrand imply that when $H \in\left(\frac{1}{2}, \frac{3}{4}\right)$,

$$
\begin{aligned}
& \lim _{T \rightarrow \infty}\left\|\frac{1}{T} \psi_{T} \otimes_{0,1} \psi_{T}\right\|_{\mathfrak{H}^{\otimes 2}}^{2} \\
& \leq \lim _{T \rightarrow \infty} \frac{2 \alpha_{H}^{2} c_{\lambda, H}^{2}}{T} \int_{[0, T]^{3}} \mathrm{~d} t_{2} \mathrm{~d} s_{1} \mathrm{~d} s_{2} \phi\left(T, s_{1}\right) \phi\left(t_{2}, s_{2}\right) \phi\left(T, t_{2}\right) \phi\left(s_{1}, s_{2}\right) \\
& =\lim _{T \rightarrow \infty} \frac{2 \alpha_{H}^{2} c_{\lambda, H}^{2}}{T^{6-8 H}} \int_{[0,1]^{3}} \mathrm{~d} t_{2} \mathrm{~d} s_{1} \mathrm{~d} s_{2} \phi\left(1, s_{1}\right) \phi\left(t_{2}, s_{2}\right) \phi\left(1, t_{2}\right) \phi\left(s_{1}, s_{2}\right) \\
& =0 .
\end{aligned}
$$

Finally, it is easy to obtain that $\psi_{T} \otimes_{1,0} \psi_{T}=f_{T} \otimes_{0,1} f_{T}$. Thus $\frac{1}{T} \psi_{T} \otimes_{1,0} \psi_{T} \rightarrow 0$ also holds as $T \rightarrow \infty$. In addition, it follows from Lemma 5.4 of web-only Appendix of Hu and Nualart (2010) that both $\frac{1}{T} \psi_{T} \otimes_{1,0} h_{T} \rightarrow 0$ and $\frac{1}{T} \psi_{T} \otimes_{0,1} h_{T} \rightarrow 0$ hold.

Proof of Theorem 1.1. Without loss of generality, we can suppose that $Z_{0}=0$. By (1.3), we obtain that

$$
\hat{\gamma}_{T}-\gamma=\sqrt{a} \frac{\frac{1}{T} X_{T}}{\frac{1}{T} \int_{0}^{T}\left|Z_{t}\right|^{2} \mathrm{~d} t}
$$

By Proposition 3.2, we need only to show $\frac{1}{T} X_{T}$ converges to zero almost surely as $T \rightarrow \infty$. Clearly, we have that

$$
\left|\frac{1}{T} X_{T}\right| \leq \frac{1}{T}\left|X_{T}-X_{n}\right|+\frac{n}{T} \frac{1}{n}\left|X_{n}\right|
$$


where $n=[T]$ is the biggest integer less than or equal to a real number $T$. Using Lemma 3.3 and since $n / T$ is bounded, we see that the second term in (3.20) goes to 0 almost surely as $T \rightarrow \infty$.

By Proposition 3.4, we see that the first term in (3.20) is bounded by $\frac{1}{T} R_{p, q} n^{q / p}$ for any $p \geq 2$ and $q>1$. Choosing $q<p$ we see that the first term in (3.20) goes to 0 as $T \rightarrow \infty$. This completes the proof of the first part of Theorem 1.1.

Now we turn to the proof of the second part. Denote $F_{T}=\frac{1}{\sqrt{T}} X_{T}$. Clearly,

$$
\bar{F}_{T}=\frac{1}{\sqrt{T}} \int_{[0, T]^{2}} e^{-\gamma(-r+s)} \mathbf{1}_{\{r \leq s\}} \mathrm{d} \zeta_{r} \mathrm{~d} \bar{\zeta}_{s}=\frac{1}{\sqrt{T}} I_{1,1}\left(h_{T}(r, s)\right) .
$$

From Theorem 1.3, Lemma 3.5 and Lemma 3.6, we see

$$
F_{T} \text { converges in law to } \zeta \sim \mathcal{N}\left(0, \frac{\sigma_{H}^{2}}{2} \mathrm{C}\right),
$$

where $\mathrm{C}$ as in Theorem 1.1. We write Equation (3.19) as

$$
\sqrt{T}\left(\hat{\gamma}_{T}-\gamma\right)=\sqrt{a} \frac{F_{T}}{\frac{1}{T} \int_{0}^{T}\left|Z_{t}\right|^{2} \mathrm{~d} t} .
$$

Therefore, it follows from the above fact, Proposition 3.2, and Slutsky's theorem that $\sqrt{T}\left(\hat{\gamma}_{T}-\gamma\right)$ converges in distribution to bivariate Gaussian law $\mathcal{N}\left(0, \frac{1}{2 d^{2} a} \mathrm{C}\right)$.

\section{Acknowledgements}

The author would like to thank an anonymous referee for very valuable suggestions which lead to the improvement of the new version.

\section{References}

M. Arató. Linear stochastic systems with constant coefficients, volume 45 of Lecture Notes in Control and Information Sciences. Springer-Verlag, Berlin (1982). ISBN 3-540-12090-4. A statistical approach.

M. Arató, A. N. Kolmogorov and Y. G. Sinai. Evaluation of the parameters of a complex stationary Gauss-Markov process. Doklady Akademii Nauk SSSR 146, 747-750 (1962).

P. Billingsley. Convergence of probability measures. John Wiley \& Sons, Inc., New York-London-Sydney (1968). MR0233396.

S. Campese. Fourth moment theorems for complex gaussian approximation. ArXiv Mathematics e-prints (2015). arXiv: 1511.00547.

Y. Chen. Product formula and independence for complex multiple wiener-ito integrals. ArXiv Mathematics e-prints (2014). arXiv: 1412.1877.

Y. Chen and G. Jiang. A note on the moment of complex Wiener-Ito integrals. ArXiv Mathematics e-prints (2017). arXiv: 1706.06032.

Y. Chen and Y. Liu. On the fourth moment theorem for complex multiple WienerItô integrals. Infin. Dimens. Anal. Quantum Probab. Relat. Top. 20 (1), 1750005, 24 (2017). MR3623878.

H. Dym and H. P. McKean. Gaussian processes, function theory, and the inverse spectral problem. Academic Press [Harcourt Brace Jovanovich, Publishers], New York-London (1976). Probability and Mathematical Statistics, Vol. 31. 
Y. Hu. Analysis on Gaussian spaces. World Scientific Publishing Co. Pte. Ltd., Hackensack, NJ (2017). ISBN 978-981-3142-17-6. MR3585910.

Y. Hu and D. Nualart. Parameter estimation for fractional Ornstein-Uhlenbeck processes. Statist. Probab. Lett. 80 (11-12), 1030-1038 (2010). MR2638974.

K. Itô. Complex multiple Wiener integral. Jap. J. Math. 22, 63-86 (1953) (1952). MR0063609.

I. Nourdin and G. Peccati. Normal approximations with Malliavin calculus, volume 192 of Cambridge Tracts in Mathematics. Cambridge University Press, Cambridge (2012). ISBN 978-1-107-01777-1. From Stein's method to universality.

D. Nualart and S. Ortiz-Latorre. Central limit theorems for multiple stochastic integrals and Malliavin calculus. Stochastic Process. Appl. 118 (4), 614-628 (2008). MR2394845. 